\title{
The influence of coefficients for the terms of a controller on generation of braking curve
}

\author{
Jan Anuszczyk ${ }^{1}$, and Andrzej Gocek ${ }^{1, *}$ \\ ${ }^{1}$ Lodz University of Technology, Institute of Electrical Engineering Systems, ul. Stefanowskiego 18/22, 90-924, Łódź, Poland
}

\begin{abstract}
This paper presents the theory about generating a braking curve and the analysis of the influence of the braking controller parameters on generation of the braking curve of the train. It has been demonstrated that the developed algorithms can be used to verify the effectiveness of the braking controller and the adjustment of the terms, and the change of these algorithms affects the shape of the generated braking curve of the train. The developed simulator cooperating with braking control system, which is reflecting the influence of braking function of the applied system used in the subway, has been analysed in terms of the impact of the parameters on the generation of braking curve. The outcomes has been presented and commented. Authors have also shown the results of the tests in the terms of an impact of a selected controller on the generation of braking curve.
\end{abstract}

\section{Theory of automatic train braking - the theoretical braking curve}

Target braking (docking) is used mainly for underground lines in order to stop the train on the platform in case the length of the train and subway platform is virtually the same. Target braking may be called an automatic process of stopping the train on the station. That kind of braking is a very important part of operating the train as it requires stopping the train on a platform of a specified length. In case of closed platforms, where entire platform is screened from the track by a wall with automatic platform-edge doors, the train stopping at metro station has to be very accurate and needs to implement the principle of "door to door" braking and is not allowed to open the marginal door beyond the platform area. Target braking of the train on the train stations should provide such siting of the train (after stopping) relative to the platform, so that all the doors of the train allow passengers to get out directly on the platform. Precise train stopping is also very important from the point of view of passengers. While the train stops in the platform the situation that the train stops in the incorrect place and a passenger could get stuck in the gap between the platform and the train cannot be allowed. Automatic braking must start at the correct distance from the stopping point and requires proper regulation of the braking force, which shall secure stopping in the required point. Target braking is one of the cases of essential braking (duty). It is basing on the gradual reduction of the actual speed of the train $V_{r z}$ to the value of allowable speed limit $V_{d}=0$, which shall be obtained at the point of restricting the speed $\mathrm{x}_{\mathrm{d}}$, without exceeding the allowable deceleration of braking $a_{h}[1,2$, 4]. Automatic target braking (docking) of the train occurs during stopping the train at the platform. Stopping should be very accurate, and the actual place of stopping the train $\mathrm{x}_{\mathrm{rz}}$ on the platform, should meet the following condition:

$$
x_{r z}=x_{d} \pm c
$$

where $c$ is the stopping tolerance.

The theoretical braking curve should take into account the desire to provide possibly high capacity of arterial railway line, it is advisable to brake with the maximal deceleration $a_{\text {hmax }}$. The limitation here is a feasible braking force $F_{h \max }$ ensuring sufficient wheels adhesion with rails. The maximum allowable braking force is a function of the actual speed of the vehicle and the profile of track and operating conditions of the line. The optimal braking process ensures gradual increase of deceleration up to a maximum value (phase I), braking with the maximum and constant deceleration (phase II) and the gradual decrease of deceleration to zero $\mathrm{V}_{\mathrm{g}}=0$ (phase III). One may distinguish two basic types of theoretical braking curve: (a) Three-sector braking curve, which takes into account I, II and III phase of braking; (b) Twosector braking curve, which takes into account I and III phase of braking. The parameters necessary to develop a braking curve are: the coordinate of a point where velocity reduction started $x_{g}$, the maximum deceleration of braking $a_{h \max }$ and the maximum value of the derivative of deceleration of braking $|\mathfrak{a}|_{\text {hmax }}$. Obtaining of an appropriate braking curve, in other words the dependency $v_{h}\left(l_{h}\right)$ may be performed substantially in three ways:

- by storing typical waveforms $v_{h}\left(l_{h}\right)$ in memory chips,

- every-time generation of braking curve using computing devices,

- every-time generation of braking curve for phase I and III and using of the recorded curve in phase II.

Figure 1 shows an example of generated theoretical curves of braking $v_{h}\left(l_{h}\right)$. Additionally for the actual speed

\footnotetext{
* Corresponding author: andrzej.gocek@gmail.com
} 
before braking process started $v_{r z}(s)$, equal to $v_{0}=80 \mathrm{~km} / \mathrm{h}$, there indicated on this curve three phases of the braking process, which were discussed above. At the point of overrunning the braking curve $v_{0}\left(l_{\text {hmax }}\right)$ there starts the process of reducing the speed to the boundary value of the speed $\mathrm{vg}_{\mathrm{g}}=0 \mathrm{~km} / \mathrm{h}$. The curve of actual speed before braking $\mathrm{V}_{\mathrm{rz}}(\mathrm{s})^{\prime}$ (marked with gray - the dottedintermittent) illustrates the case of starting braking from $\mathrm{v}^{\prime}=40 \mathrm{~km} / \mathrm{h}$. Due to the allocation of the setup for generation of braking curve one may distinguish two groups of solutions:

- a generation in the vehicle with the transmission to the vehicle of output data for calculations, or

- a generation by the trackside equipment with the transmission of the results of calculations to the vehicle.

The choice of the location for the setup for generating braking curve depends on the range of automation of driving the vehicle, and especially depends on the amount of transmitted information and the type of data transmission equipment. It should be emphasized that the working conditions of the data transmission system between the track and the vehicle are very difficult, especially due to strong interference in the transmission channel. Hence the need to strive to minimize the required number of transmitted information $[1,2,4]$.

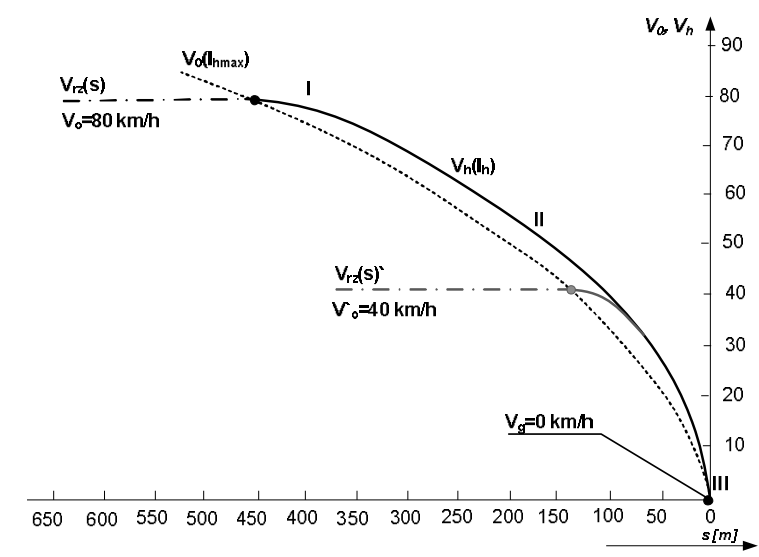

Fig.1. The theoretical curves of braking and braking start for the speed of braking start $\mathrm{v}_{0}=80$ and $40 \mathrm{~km} / \mathrm{h}$.

\subsection{Generation method of braking curve}

The primary task of a brake control system is to obtain in the point of speed restriction $x_{d}$, permissible speed $V_{d}$ with certain restrictions. To conduct this process the following data have to be obtained: constant parameters - capacity of electrodynamic and mechanical braking $a_{h}$, coordinates of the stopping points $x_{z}$, vertical and horizontal line profile $m_{z p}$ given in promilles and variable parameters actual train speed $V_{r z}$, actual train stopping position $x_{r z}$, permissible speed $V_{d}$, train load and any external interferences $z$ - also including elementary resistance to motion. The executing system has to perform the following tasks (in the given order):

1) determine $x_{0}$ point for starting the braking process,

2) determine the actual train position $x_{r z}$ and begin to measure the actual train speed $V_{r z}$,
3) calculate the braking speed $V_{h}$,

4) calculate in real time the braking force $F_{h}$ and control the braking process.

The steps from enumeration 1-3 are measurement and computing operations. Therefore, these steps are not affected by the integrated train braking system.

The above-mentioned actions in no. 4 have a controlexecution nature and are related to the integrated braking system $[1,2,4]$. The structure of this system is shown in Fig. 2. The above-mentioned calculation structure may be easily calculated in a generic way in relation to a simplified computational model, and consists of the following tasks: (a) determination of initial braking point $x_{0},(b)$ calculation of the distance $l_{h}$ - between the front of the train $x_{r z}$ and the point of speed restriction $x_{d},(c)$ generation of the theoretical braking curve $V_{h}\left(l_{h}\right)$.

The tasks of the control-execution structure are based on the calculation of the difference $\Delta v$ between braking speed $V_{h}$ and the actual train speed $V_{r z}$, next the determination of the braking force $F_{h}$ and the execution of the braking process (electrodynamic and mechanical braking phases). Braking devices are implementing the braking process with a specified value of braking deceleration $a_{h}$. The parameters which are permanently supplied to the system are dependent on the type of the train, applied the braking system and the weight of the train. Actual speed $V_{r z}\left(x_{r z}, t\right)$ is a function of the place where the train is located $x_{r z}$ and time $t$.

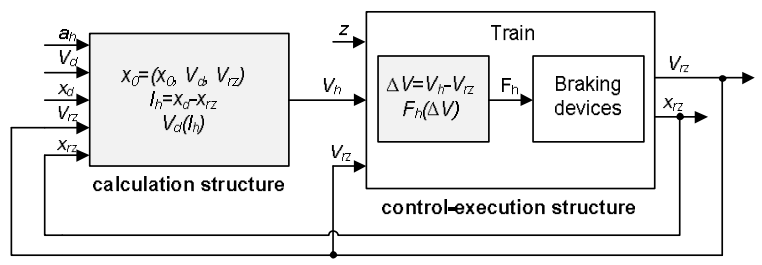

Fig. 2. Structure of the automatic braking system.

The place where the train is positioned on the track $x_{r z}(t)$ is a function of time. The train is the source of the values mentioned above. The allowed speed depends on the situation of the movement and the parameters of the rail setup on the metro line, and it depends on the location of points of speed restriction $x_{g}$ on the metro line as well as on the time $t$. The source of this value is trackside equipment related to railway traffic control devices. The points of restrictions (speed change) are constant in time. The parameters required to calculate the theoretical curve of braking depend on the speed of the braking $V_{h}\left(l_{h}\right)$, which is affected by the actual - current distance between the vehicle and the point of speed limitation $l_{h}=x_{g}-x_{h}$ and deceleration $a_{\text {hmax }}$. The starting point of braking is dependent on the actual speed of the train $V_{r z}$, terminal speed $V_{g}$ and technical capacity of the braking train. In this step, it becomes necessary to take into account the load of the train, which is strictly dependent on the load caused by passengers. For the proper implementation of target braking the control-executive system requires provisioning of the curve of braking speed $V_{h}\left(l_{h}\right)$, and the actual speed of the train $V_{r z}\left(x_{r z}, t\right)$. The signal responsible for the implementation of the braking process is the 
braking force $F_{h}$, dependent on the braking speed $V_{h}\left(l_{h}\right)$ and the actual speed $V_{r z}\left(x_{r z}, t\right)$.

\section{Simulation studies of the selected cases}

\subsection{Methodology of determining the braking precision}

The outcome of the created simulator relies on the implementation of the controlling system, which corresponds to a layout used in the underground to generate docking stage, it takes into account [1]: the train movement equations, profile $i$, resistance to motion $W$, the combination of the traction characteristics $F_{\text {ham }}(v)$, delay of the controller, line spacing, wire loops lengths, wire loops crossings, permitted line speed, restriction of increase of braking deceleration, the derivative of acceleration with respect to time, weight of the train, train filling and electrical controlling signals used in the process of precision braking.

The simulation model communicates with a braking controller, which is based on a discrete PID regulator, in a connection time $t_{k o m}=50 \mathrm{~ms}$. The braking controller reflects the operations of the on-board device embeddable on a real underground train, which cooperates with the system which realizes docking.

Depending on the signal from the controller, the simulator implements one of the available driving modes (steady ride, free run or braking). Next, the program checks the initial parameters of train physics, line profiles, the drive characteristics ( $F_{\text {ham }}$ - the braking force of traction machines), parameters of braking system, train weight, etc. The algorithm of the software detects the number of a wire loop (loop detection) and sets the value of the distance to the stopping point. Depending on the selected driving mode, the script performs calculations of resistance to motion, profile and the distance during braking. The calculations are performed with a time step $t_{h}=t_{h}+\Delta t$, to a train stop $(v=0)$. The value of the total braking force $F_{h}=F_{\text {ham }}+W$ is being calculated during the braking process. For the following steps, the program calculates the actual braking distance $s$, speed $v$ and the actual deceleration $a_{h}$ on the basis of total braking force $F_{h}$, resistance of motion $W$ and profile $i$. The actual train speed $v$ and the distance $s$ are the input data supplied to the regulator. The functional scheme of this process is shown in the Figure 3.

\subsection{The influence of coefficients for the PID terms of a controller on generation of braking curve}

The description of the authorial tool developed to perform the objective research and the applied target braking steering systems of the train with AC and DC drive were extensively discussed in papers $[1,2,4]$. The developed simulator cooperating with braking control system PID, which is reflecting the influence of braking function of the applied system used in the subway, has been analysed in terms of the impact of the parameters on the generation of braking curve. In particular, the effect of changing parameters of the terms of a controller to offset of the braking distance was analysed.

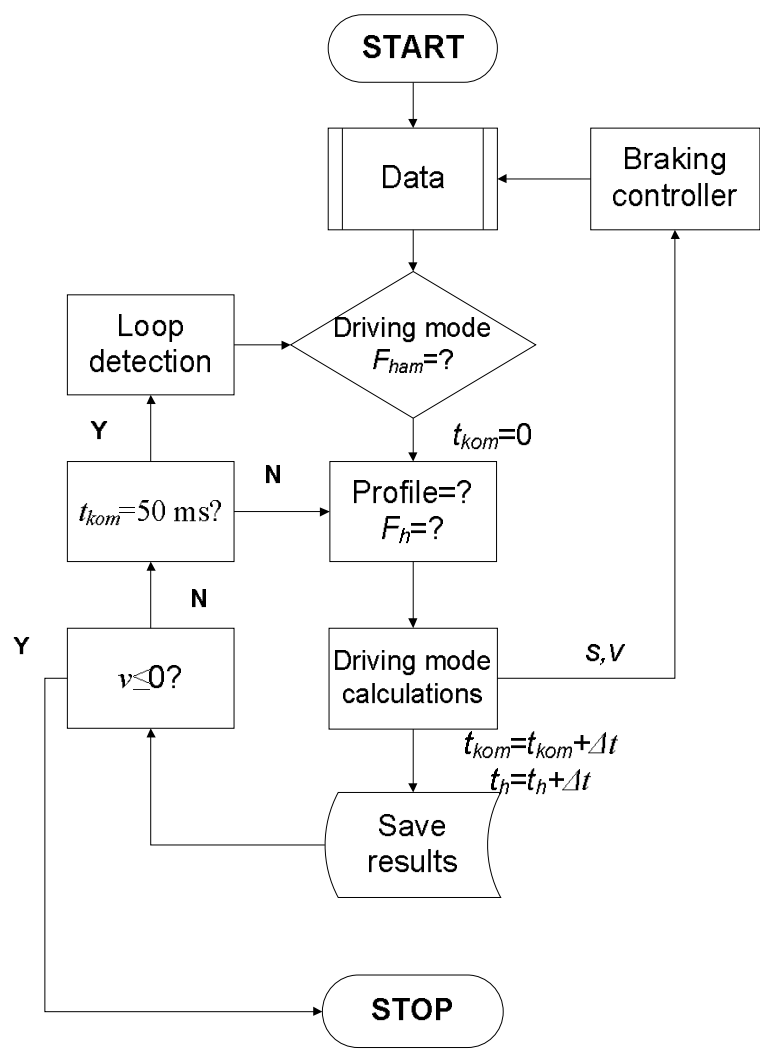

Fig. 3. A general block diagram of the simulation model functional scheme.

For this purpose four sets with values of terms of parameters of controller were tested $-K_{p}, T_{i}, T_{d}$. Considering the proposed sets of terms of parameters of the controller, using the developed target braking simulator, the calculations were carried out for several theoretical rides of a train, for which an infringe of braking path was scheduled. The results of calculations are presented in Table 1, where the accuracy of stopping (Dham) is given from the point of required stop. In a situation where the train passes by a stopping point the result has the sign "minus".

Table 1. The results of calculation of an infringe of braking path of a train for different controller parameters.

\begin{tabular}{|c|c|c|c|c|}
\hline & Set 1 & Set 2 & Set 3 & Set 4 \\
\hline $\begin{array}{c}\text { The accuracy of } \\
\text { stopping the train } \\
\text { Dham (infringe of } \\
\text { braking path) [m] }\end{array}$ & $-1,10$ & $-3,41$ & 1,34 & 0,38 \\
\hline
\end{tabular}




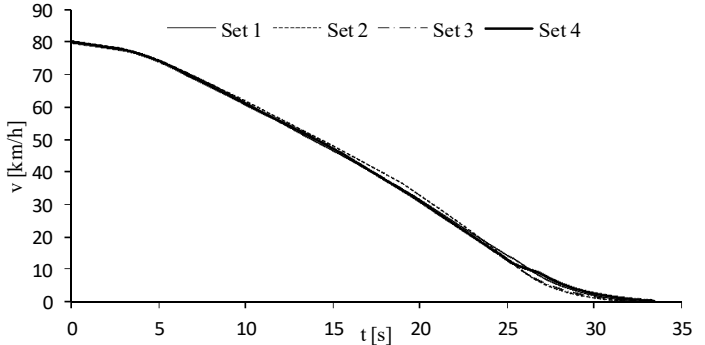

Fig. 4. Trajectories of braking speed depending on the adopted controller parameters (parameters of terms of controller); the degree of filling $s t=1,0$; speed of initiation of braking $\mathrm{v}_{\mathrm{p}}=80$ $\mathrm{km} / \mathrm{h}$, the complex route profile.

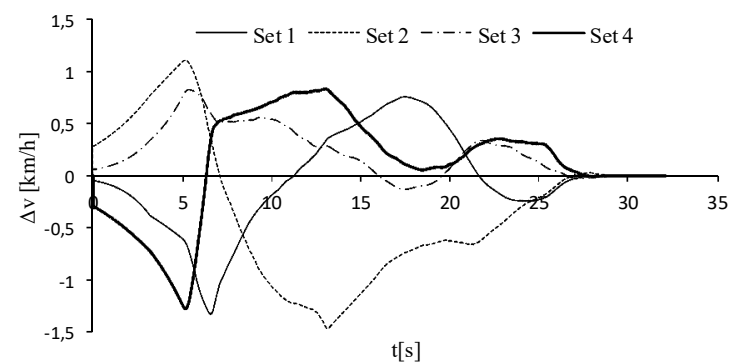

Fig. 5. The suppressed oscillatory course for adopted sets of terms of controller parameters; the degree of filling $s t=1,0$; speed of initiation of braking $\mathrm{v}_{\mathrm{p}}=80 \mathrm{~km} / \mathrm{h}$, the complex route profile.

After discerning literature in this field $[3,5,6,7,8,9$, $10]$ a deficiency has been found, to expand the research of the influence of coefficients for the terms of a controller on generation of braking curve, the authors have considered two types of implemented controller - PID and PI, using as a base the set 4 of the parameters of terms of controller. Results are presented below in the form of 3D graphs.

PI

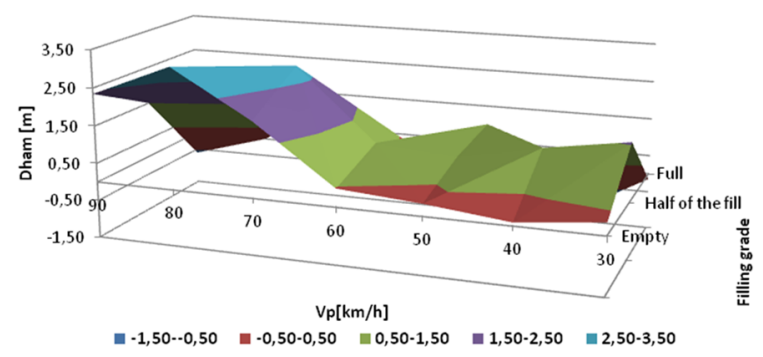

Fig. 6. Simulation for PI controller $\left(T_{d}=0\right), T_{p}=100 \mathrm{~ms}$, speed of initiation of braking $-\mathrm{v}_{\mathrm{p}}$, the degree of filling the train by passengers - filling grade, accuracy of stopping $-\mathrm{D}_{\text {ham }}$.

Performed simulations show that a PI controller allows to improve static accuracy in range of medium and low speeds $(30-60 \mathrm{~km} / \mathrm{h})$, for speeds above $60 \mathrm{~km} / \mathrm{h}$, braking accuracy is considerably reduced (comparing with outcomes for PID controller shown in the Figure 7). So it is justified to use a PID controller, this type of controller is typically used for objects subjected to rapidchange disturbances, what occurs in the braking process, and PID controller allows to shorten the adjustment time.

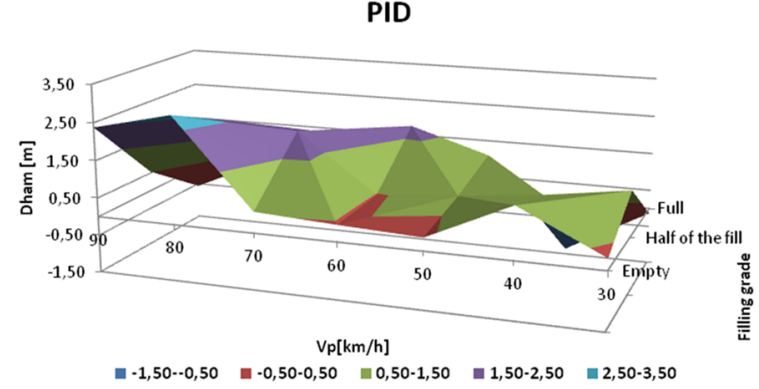

Fig. 7. Simulation for PID controller (parameters of terms of controller - set 4 ), $T_{p}=100 \mathrm{~ms}$, speed of initiation of braking $\mathrm{V}_{\mathrm{p}}$, the degree of filling the train by passengers - filling grade, accuracy of stopping - Dham.

PI controllers allow for the elimination of slowmoving disturbances, which translates into a zero deflection fixed and a longer regulation time. The PID controller enables shorter times of duplication than PI regulator without fear of the formation of oscillation in the closed system (shown in the Figure 5), so it eliminates the effects of interference more quickly.

\section{Conclusions}

The developed simulator $[1,2,4]$ enables modelling the shape of the computational curve of braking speed, through the ability to change electrical parameters of the controller operating in the system of target braking, which is illustrated by characteristics shown in Figure 4.

As it can be seen in Figure 5, which presents the trajectories of braking curve $\mathrm{f}(\mathrm{t})=\Delta \mathrm{v}$ during the braking process, the smallest oscillations of the concerned sets of terms of parameters of regulator are for the Set 4. All trajectories are characterized by suppressed oscillations. From simulation calculations it may be noticed that the change of terms of parameters of a controller affects the course of target braking process, and consequently the value of the infringe of the train's braking path.

As the results show, the PID controller enables better stopping accuracy than a PI controller. The developed simulator [1] cooperating with the brake adjustment block can be used to study the influence of electrical parameters on the process of target braking and may be used for selection and verification of parameters for braking controller. The analysis deduces that changing the electrical parameters of the regulator affects the process of target braking, and consequently influences the value of infringe of the braking path of the train and that the developed algorithms can be used to verify the effectiveness of the braking regulator.

\section{References}

1. Anuszczyk J., Gocek A.: The influence of operating parameters on braking precision of metro trains. Conference Proceedings of XII International Conference MET2015 Modern Electrified Transport, Trogir, Croatia, (2015), pp. 1-7. 
2. Barański S., Gocek A., Steczek M.: The computer modeling of target braking of underground trains taking into account different types of traction drives. Logistics - the science Vol. 4/2015, (2015), (in polish).

3. Domínguez M., Fernández-Cardador A., Cucala A. P., Pecharromán R. R.: Energy Savings in Metropolitan Railway Substations Through Regenerative Energy Recovery and Optimal Design of ATO Speed Profiles. IEEE Transactions on Automation Science and Engineering, Vol. 9, No 3, July, (2012).

4. Gocek A., Steczek M.: Simulation studies of target braking accuracy of an underground train driven by an AC motor. Technical Magazine, Electrical Issue 1-E (2), pp. 67-77, (2016).

5. Gao Bing, Dong Hairong, and Zhang Yanxin: Speed Adjustment Braking of Automatic Train Operation System Based on Fuzzy-PID Switching Control. 2009 Sixth International Conference on Fuzzy Systems and Knowledge Discovery. China, (2009).
6. Kawashima H., Komura F., Honma K., Iwasaki A., Takaoka T., Tashiro K., Monma N.: A Total Railway System Simulator and its Application to the Evaluation of Energy Saving Train Operation. Wessex Institute of Technology Conference, COMPRAIL 92, Washington, (1992).

7. Mincardi R., Savio S., Sciutto G.: Models and tools for simulation and analysis of metrorail transit systems. COMPRAIL 94. Italy, (1994).

8. Pniewska B.: Follow-up control of the traction vehicle at the scheduled timetable. Phd Thesis. Technical University of Warsaw, Warsaw, (1995), (in polish).

9. Zhao N., Roberts C., Hillmansen S., Western P., Chen L., Tian Z., Xin T., Su S.: Train Trajectory Optimization of ATO Systems for Metro Lines. 2014 IEEE 17th International Conference on Intelligent Transportation Systems (ITSC). Qingdao, China, (2014).

10. Zhu X., Liu X.: The Modelling of Test Systems of Automatic Train Operation (ATO) in Urban Rail Transit Based on LABVIEW. International Conference on Computer Application and System Modelling (ICCASM 2010). China, (2010). 